\title{
AIDS-Related Anal Canal Carcinoma
}

National Cancer Institute

\section{Source}

National Cancer Institute. AIDS-Related Anal Canal Carcinoma. NCI Thesaurus. Code C133095.

A carcinoma arising from the anal canal and occurring in HIV-positive patients. 